\title{
Restricted Hilbert Transform for Non-Hermitian Management of Fields
}

\author{
W.W. Ahmed®, ${ }^{1, *}$ R. Herrero, ${ }^{2}$ M. Botey, ${ }^{2}$ Y. Wu,${ }^{1, \dagger}$ and K. Staliunas ${ }^{2,3,4,+}$ \\ ${ }^{1}$ Division of Computer, Electrical and Mathematical Sciences and Engineering, King Abdullah University of \\ Science and Technology (KAUST), Thuwal 23955-6900, Saudi Arabia \\ ${ }^{2}$ Departament de Física, Universitat Politècnica de Catalunya (UPC), Colom 11, E-08222 Terrassa, Barcelona, \\ Spain \\ ${ }^{3}$ Institució Catalana de Recerca i Estudis Avançats (ICREA), Passeig Lluís Companys 23, E-08010 Barcelona, \\ Spain \\ ${ }^{4}$ Vilnius University, Laser Research Center, Saulètekio al. 10, Vilnius, Lithuania
}

(Received 4 May 2020; revised 17 July 2020; accepted 26 August 2020; published 7 October 2020)

\begin{abstract}
Non-Hermitian systems exploiting the synergy between the properties of closed-conservative systems and open-dissipative (gain-loss) systems have recently become the playground to uncover unusual physical phenomena. Indeed, the spatial symmetry breaking in such systems allows tailoring of the wave propagation at will. Inspired by such a property, we propose a feasible approach based on local Hilbert transform to control the field flows in two- or higher-dimensional non-Hermitian systems. Moreover, we invent an iterative procedure to reduce the dimensionality of complex refractive-index parameter space to two, one, or zero dimensions, restricting the complex refractive index within practical limits. The proposal provides a flexible way to systematically design locally non-Hermitian systems realizable with a limited collection of realistic materials.
\end{abstract}

DOI: 10.1103/PhysRevApplied.14.044010

\section{INTRODUCTION}

Physical causality is closely related with the celebrated Hilbert transform (HT). In optics, the HT is well known as the Kramers-Kronig (KK) relation, connecting the real and imaginary parts of the spectrum of the response function of a system [1] in a way that it ensures that response must occur at a later (not earlier) time of its excitation. Such a HT breaks the time symmetry and accounts for the "invisibility" of the future.

Recently, an analogous HT was proposed in space to define the spatial profile of the complex susceptibility of a system to non-Hermitically engineer a nonisotropic response, thus breaking the spatial symmetry. In one dimension, space and time are both scalars, and therefore, the HT in space and time are mathematically equivalent [2]. The invisibility of the future or the absence of the flow of information to the past may be regarded as analogous to the absence of scattering, say backscattering irradiating in the direction opposite to illumination [3-5]. In two and three dimensions, however, the situation becomes more engaged as backscattering (unidirectional invisibility) can be eliminated from different directions.

\footnotetext{
*waqas.waseem291@gmail.com

†ying.wu@kaust.edu.sa

+kestutis.staliunas@icrea.cat
}

For instance, in a two-dimensional (2D) space, it is possible to manipulate the scattering of an object towards particular directions $[6,7]$ by correspondingly modifying its spatial susceptibility function via the HT.

The spatial symmetry breaking of the system response, on the global scale, is extensively studied in the context of parity-time (PT) symmetry to explore physical effects [8-18]. Moreover, such symmetry breaking of the spatial response may occur locally, i.e., being different at different spatial positions, which allows engineering of complex directionality fields, with particular topologies, such as axisymmetry [19], or following arbitrary vector fields being the background potential from periodic and quasiperiodic to random [20].

Despite some attempts [7,21], a practical realization of the HT in space, in either a global or local sense, remains a great challenge. Actual realizations of the spatial HT are severely restricted by the limited available materials that can satisfy the required complex refractive-index requirements $\left(n_{\mathrm{Re}}, n_{\mathrm{Im}}\right)$. With the development of metamaterials, the response function of which relies on their subwavelength artificial structures, rather than on chemical compositions, the possible regime of the refractiveindex profile is greatly expanded. Thus, metamaterials bring about the opportunity to realize a precise relation between the real and imaginary parts of the response function required by the spatial HT. For instance, metamaterials based on a particular collection of "metachips," satisfying 
a given response spectra, allow a spatial HT profile in the microwave regime [22]. However, it is not possible to realize arbitrary relations between real and imaginary parts of susceptibility from a limited collection of real materials. In semiconductor microstructures, the situation is also similar, as actual active materials have a specific relation between index and gain-loss $\left(n_{\mathrm{Re}}, n_{\mathrm{Im}}\right)$.

The fundamental question that arises is if an actual field of unidirectionality can be implemented, with either a global or local topology, using the HT approach within restricted (realistic) range of complex susceptibility of a material. Arbitrary unidirectional vector fields can be designed by locally modifying the complex susceptibility (or complex refractive index) using the local Hilbert transform. As an example, Fig. 1 illustrates such a directionality field consisting of a sink and a source. However, the resultant complex index profile demands a large number of actual materials for practical realization due to the continuous variation of complex index values in space [see Fig. 1(b) and 1(c)]. To resolve this issue, we propose to introduce restrictions for the complex index values, constraining them around certain particular values or confining them in some particular areas of the parameter space $\left(n_{\mathrm{Re}}, n_{\mathrm{Im}}\right)$. Such a restriction possibly may not limit the field unidirectionality or total invisibility, resulting in a basic scattering reduction. We propose an iterative procedure to include such constraints to design the locally PT-symmetric systems with realistic index and gain-loss values. (a)

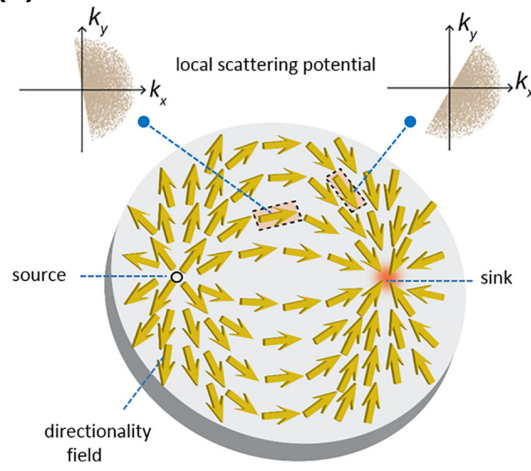

(b)

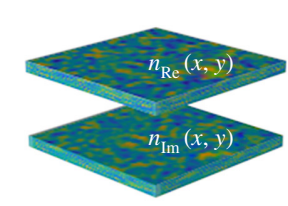

(c)

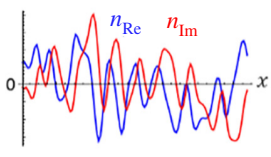

FIG. 1. Locally modified non-Hermitian medium providing the Hilbert transform. (a) Scattering potential of the medium, $n_{\mathrm{Re}}(x, y)$, modified by local HT to mold the flow of light in the desired direction. Here, the optical medium is specifically tailored to create source and sink fields, which involves gainloss regions, $n_{\mathrm{Im}}(x, y)$. The generated two-dimensional complex potential, $n(x, y)=n_{\mathrm{Re}}+i n_{\mathrm{Im}}$, provides spatially dependent directionality in the neighborhood of each spatial point to control field flows. (b) 2D complex refractive-index profiles of the modified HT medium, requiring a large number of materials corresponding to different spatial points. (c) Cross-section profile of the complex index.

\section{DIMENSIONALITY RESTRICTIONS}

Typical restrictions of non-Hermitian media to engineer the local HT considered in our study are as follows:

(1) HT media as a mixture of two different materials with different complex refraction indices. Restricting the total filling factor of two materials leads to a onedimensional (1D) manifold in the refractive index in complex refraction-index parameter space, while limiting the total filling factor of two materials to some upper bound results in a general 2D manifold of available complex refraction indices, a surface [Fig. 2(a)]. Mixing of chemical components and/or manipulating porosity is a common practice to vary the refraction indices in desired range, typically between 1.2 and 2.1 in vapor-deposition techniques [23,24].

(2) HT media as metamaterials built from a continuous family of size-scaled metachips in the microwave range [22], which restricts the complex refraction-index space to a ring [Fig. 2(b)].

(3) HT media as a "poor man's" collection of discretely size-scaled resonators (for instance, of Helmholtz resonators in acoustics or microwave metachips), corresponding to a discrete set of points in the complex index space [Fig. 2(c)].

In this way, to show the universality of the method to engineer the desired HT profiles in the restricted parameter space, we analyze these distinct cases, which entails

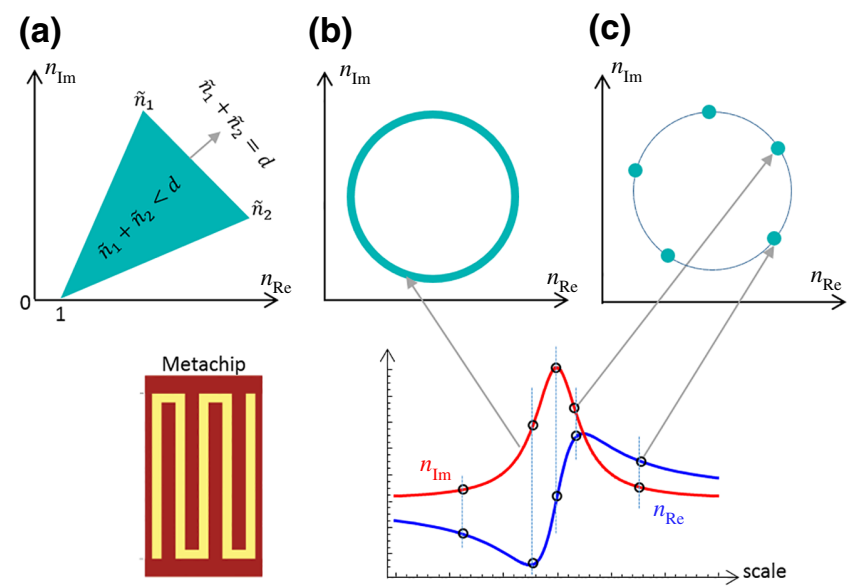

FIG. 2. Restrictions of the local HT for different restriction dimensionalities. (a) Mixture of two different materials with $\tilde{n}_{1}$, $\tilde{n}_{2}$; for instance, fixing the total density $\tilde{n}_{1}+\tilde{n}_{2}=d$ (solid or liquidlike) gives a line, and a mixture of two materials restricting the maximal density, $\tilde{n}_{1}+\tilde{n}_{2}<d$, gives an area in complex space. (b) Continuum of scalable metachips gives an available ring in $n$-complex space. (c) Limited collection of scaled-in-size metaatoms gives a set of points in complex space (five points in this case). 
reducing the dimensionality in parameter space of complex refractive indices. For instance, Fig. 2 illustrates the kind of dimensionality reductions of the three proposed restrictions: (a) $2 \mathrm{D} \rightarrow 2 \mathrm{D}$, (b) $2 \mathrm{D} \rightarrow 1 \mathrm{D}$, and (c) $2 \mathrm{D} \rightarrow 0 \mathrm{D}$.

\section{RESULTS}

\section{A. Restricted Hilbert transform}

To implement the proposed schemes, we apply an iterative procedure. First, we perform a local HT on an arbitrary background potential to create desired directionality flows, such as a sink, which require a large area of real and imaginary values of $n(\vec{r})$. Second, we shift the unallowed values of a complex of $n(\vec{r})$ to the restriction area. As this process partially breaks the HT, then we perform the HT again and repeat the procedure. We repeat this iterative procedure until the index values converge. We define a correlation coefficient for the generated vectorial potentials with different restriction dimensionalities to characterize the accuracy and convergence of the Restricted HT, which confirms that this iterative approach leads to converging results in all cases studied. To verify the directionality effect in Restricted HT, we perform numerical simulations using the Schrödinger equation (for paraxial optics) for linear systems with given complex vectorial potentials. We also perform full-wave simulations to demonstrate the functionality of the proposal beyond the paraxial approximation (see the Supplemental Material [25]). (a)

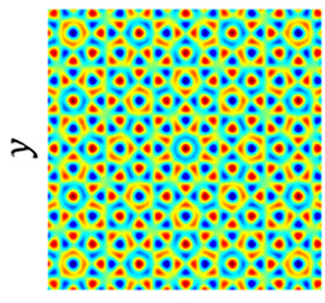

$x$

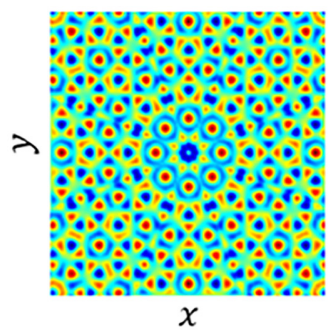

$x$

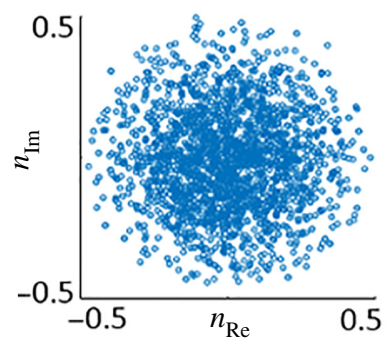

(b)

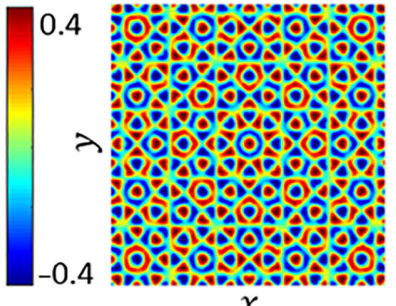

$x$
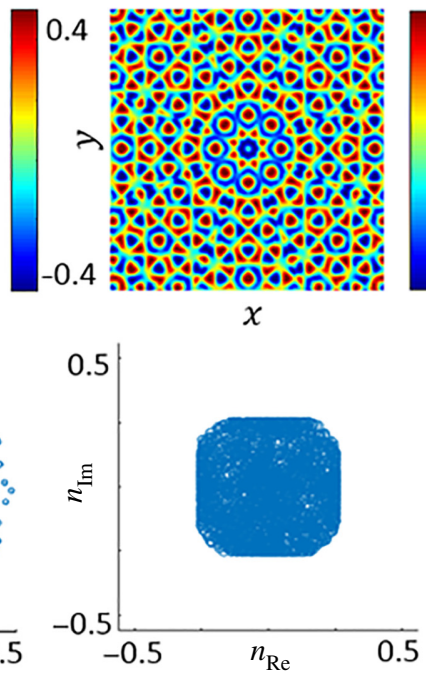

(c)

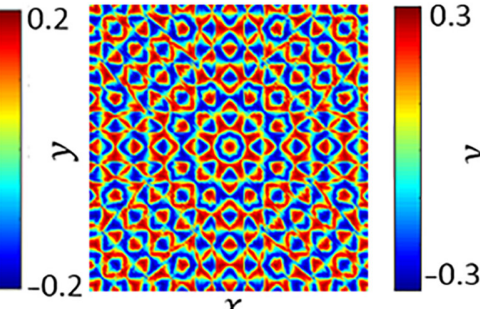

(d)

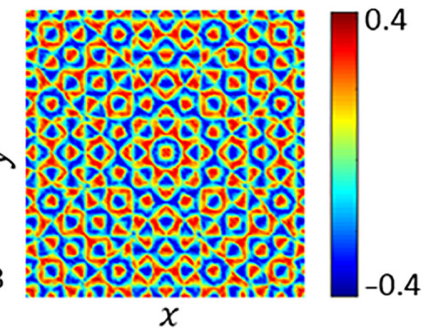

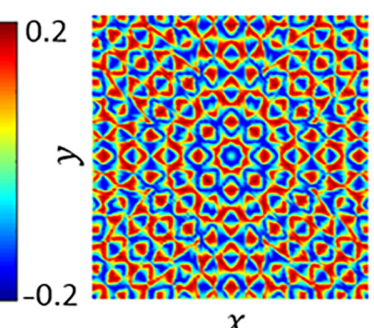

$x$

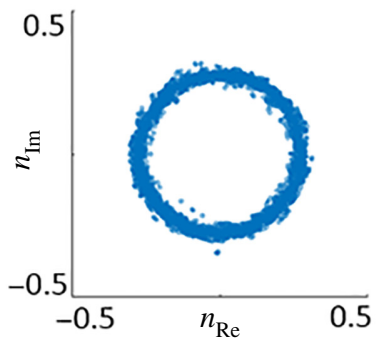

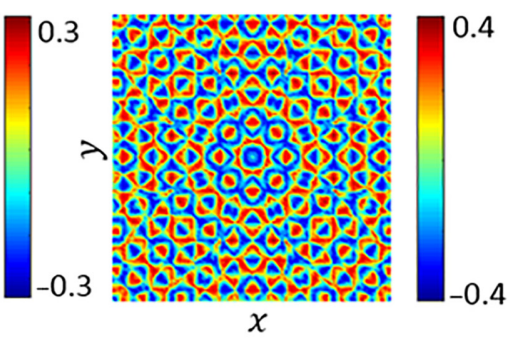

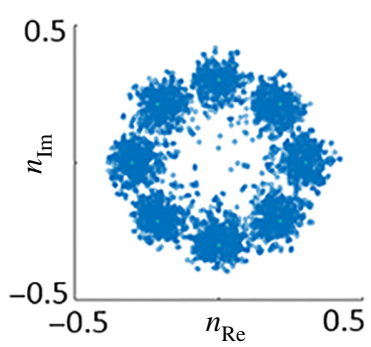

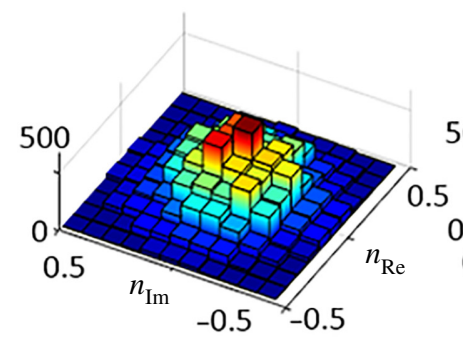
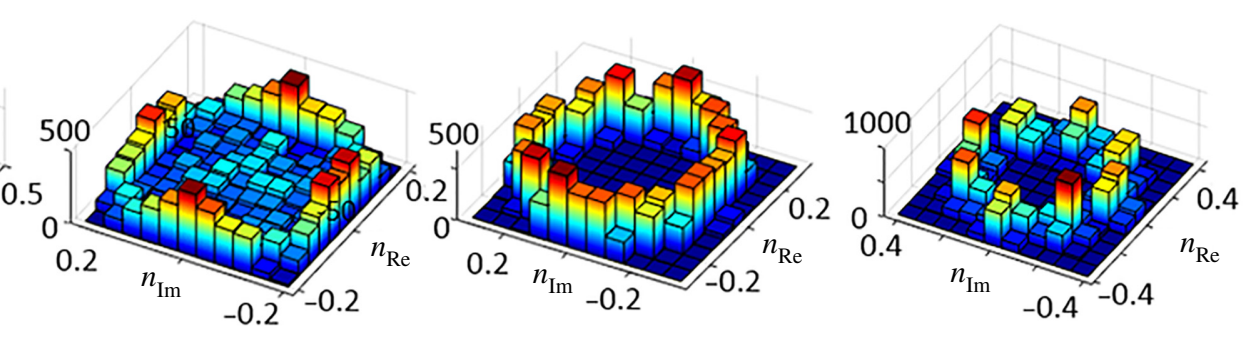

FIG. 3. Complex refractive-index distributions for sink directionality: (a) local HT, (b)-(d) Restricted local HT with hexagonal background pattern. In Restricted HT cases, the complex index profiles are obtained after 15 iterations. First row presents the real part of refractive index, second row depicts the imaginary part obtained from the local HT, and third and fourth rows depict the corresponding restricted index values and density distribution in complex space, respectively. Size of computation region is $32 \lambda_{0} \times$ $32 \lambda_{0}$ and generated distributions have spatial resolution of about $0.25 \lambda_{0}$ with wavelength $\lambda_{0}$. 
As an example of a possible realization of a Restricted HT media, we consider an initial real-valued hexagonal profile, $n_{\mathrm{Re}}(\vec{r})$ (see top-left panel in Fig. 3), and generate the corresponding gain-loss profile, $n_{\operatorname{Im}}(\vec{r})$, by applying the local HT [20] to ensure the converging directionality field in the form of a sink: $p(\vec{r})=-\vec{r} /|\vec{r}|$. The parameter space of complex refractive index $\left(n_{\mathrm{Re}}, n_{\mathrm{Im}}\right)$ provides different material parameters as functions of the spatial location [see Fig. 3(a)]. To realize such complex profiles with available materials, we apply a local HT iteratively to restrain complex index values within physical limits. The results for different restriction dimensionalities are shown in Figs. $3(\mathrm{~b})-3(\mathrm{~d})$. The index values in complex space $\left(n_{\mathrm{Re}}, n_{\mathrm{Im}}\right)$ illustrate that the iterative procedure precisely limits the refractive indices within the designated area, ring, or a set of points on a ring (see the third row of Fig. 3). The corresponding density distributions of a complex index, plotted in the last row of Fig. 3, also show the spreading of restricted values inside the desired bounds. Notably, the Restricted HT provides many possible ways to restrict the complex indices during the iterative procedure and some of them are discussed in the Supplemental Material [25]. The procedure is independent of the background potential profile and can be applied to an arbitrary initial distribution, such as quasiperiodic, random, and localized. We note the shift of the restriction area in the complex index space is possible in such linear systems. Moreover, an iterative procedure can be applied to exclude the gain to achieve analogous directionality fields with only losses, i.e., constraining the HT to a restricted parameter space, thus increasing the feasibility for practical realizations (see the Supplemental Material for random background medium and only lossy materials [25]).

\section{B. Convergence of Restricted Hilbert transform}

To analyze the robustness and convergence of the iterative procedure, we determine the correlation coefficients in terms of flow of complex potential generated by the HT under different restrictions. We define the correlation coefficient as $C=\int p(\vec{r}) f_{k}(\vec{r}) d r / \sqrt{|p(\vec{r})|^{2} d r^{2}\left|f_{k}(\vec{r})\right|^{2} d r^{2}}$, where $p(\vec{r})$ is the reference flow and $f_{k}(\vec{r})=i\left(U_{k} \nabla U_{k}^{*}-U_{k}^{*} \nabla U_{k}\right)$ is the potential flow after $k$ iterations with restrictions. For the cases shown in Fig. 3, we assume sink directionality to generate the complex vectorial potentials. Therefore, the reference flow maintains the form of the sink, $p(\vec{r})=-\vec{r} /|\vec{r}|$, to determine the correlation coefficient.

We expect that the constraints will have a weak influence on the pattern of potential flows with reference to the sink. Here, we compute two correlation coefficients, namely, $C_{0}$ and $C_{1}$, for which $f_{k}(\vec{r})$ is calculated from a complex refractive distribution obtained by applying HT before and after the restriction at $k$ iterations, respectively. The difference between $C_{0}$ and $C_{1}$ estimates the accuracy of the proposed Restricted HT. To illustrate the difference of applying the HT before and after restrictions, we present the refractive indices in complex space after the 1st and 12th iterations in Figs. 4(a)-4(c). The second row in Figs. 4(a)-4(c) shows the convergence of values of refractive indices in complex space for all cases. In Fig. 4(a), the Restricted HT accurately limits the complex refractive indices over the desired ranges. However, for Figs. 4(b) and 4(c), the refractive indices spread around the ring and the chosen discrete points. This spreading behavior, due to restrictions in complex space, may be associated with the uncertainty principle, i.e., the smaller the restricted area, the larger the spreading. The correlation coefficients $C_{0}$ and $C_{1}$, which are calculated during the iterative procedure, are plotted in Fig. 4(d). In the square case, we obtain exactly the same values for both correlation coefficients after 15 iterations, ensuring 100\% accuracy. However, we
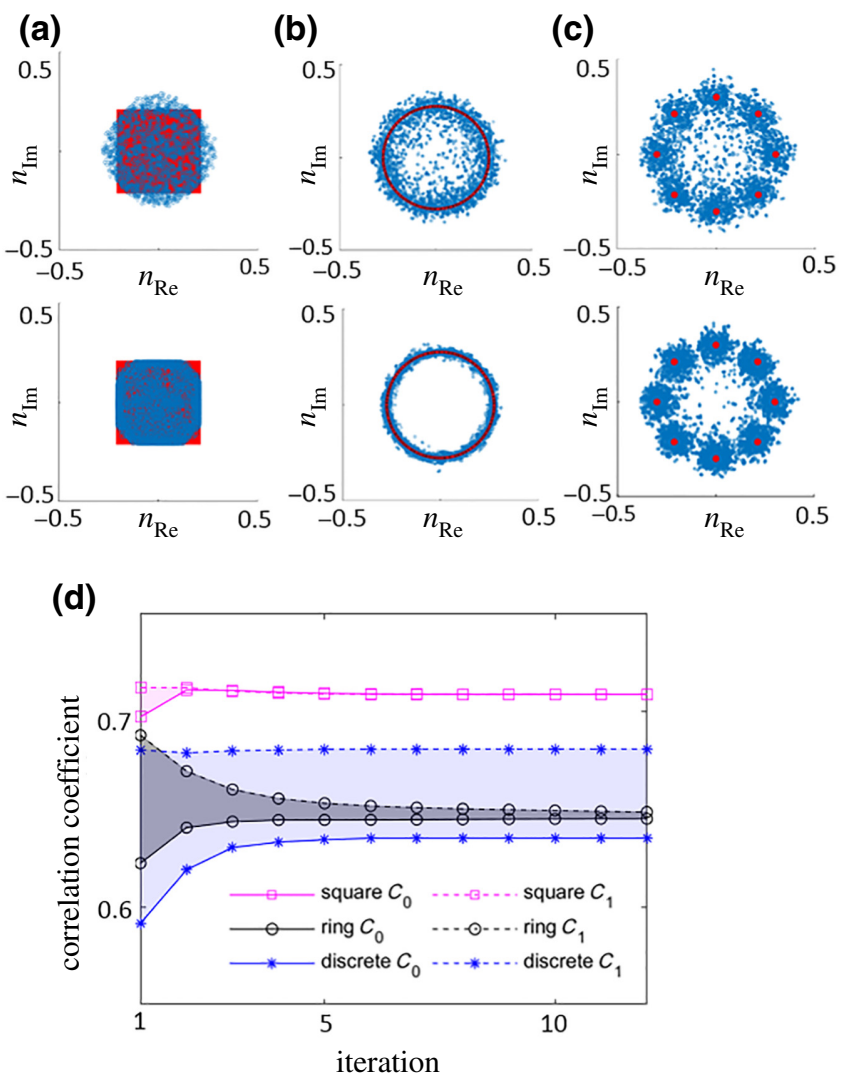

FIG. 4. Convergence of Restricted local Hilbert transform. (a)-(c) First and second rows depict refractive indices in complex space after 1st and 12th iterations, respectively. Red and blue colors illustrate the desired and computed restricted complex refractive-index values obtained from local HT, respectively. (d) Correlation coefficients $\left(C_{0}\right.$ corresponds to solid line; $C_{1}$ corresponds to dotted line) in terms of flow of the complex potential generated by HT during iterative procedures for different restriction dimensionalities: $\square, 2 \mathrm{D} \rightarrow 2 \mathrm{D}$; $\circ, 2 \mathrm{D} \rightarrow 1 \mathrm{D}$; $*, 2 \mathrm{D} \rightarrow 0 \mathrm{D}$. 
find about $1 \%$ and $5 \%$ difference in correlation coefficients for ring and discrete cases, respectively. Notably, the difference between correlation coefficients, for the considered restrictions, remains the same, regardless of background pattern, but the exact correlation values, $C_{0}$ and $C_{1}$, depend on the background pattern. For instance, in a purely sinusoidal pattern, these values are close to one, but lower than unity in the considered hexagonal pattern. We note that the convergence of correlation coefficients requires more iterations when the restriction area shrinks. In the square case, the large restriction area leads to a fast convergence, as compared with the ring and discrete cases. However, the iterative procedure converges after ten iterations in all cases, as depicted in Fig. 4(d).

(a) (i)

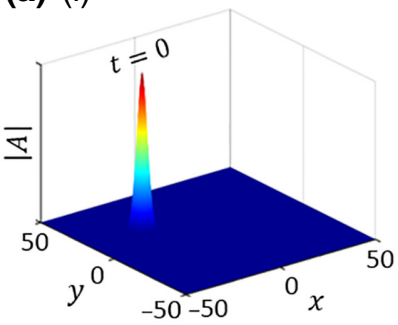

(ii)

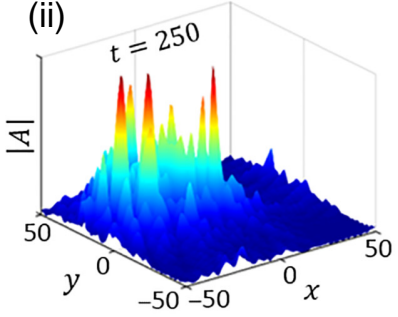

(iii)

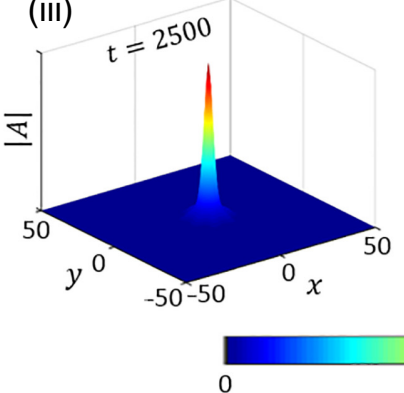

FIG. 5. Field evolution in modified HT media for sink directionality. System is excited with a Gaussian beam placed at an arbitrary position within the structure as shown in (ai). Numerically calculated transient and final localized states are shown in (aii) and (aiii), respectively. Steady-state distributions for restricted cases are (b) a square area, (c) ring, and (d) a set of discrete points on the ring. Results indicate that a Gaussian source, initially located at an arbitrary position, localizes around the center in all cases after sufficient propagation time.

\section{Field evolution in Restricted HT media}

To validate the "Restricted Hilbert transform" proposal, we perform numerical simulations using a paraxial equation of diffraction (equivalent to the Schrodinger equation for a quantum wave function) expressed in the form

$$
\partial_{t} A(\vec{r}, t)=+i \nabla^{2} A(\vec{r}, t)+i U(\vec{r}) A(\vec{r}, t),
$$

where $A(\vec{r}, t)$ is a slowly varying complex field-enveloped distribution in space $\vec{r}(x, y)$ and evolving in time $t . U(\vec{r})=$ $n_{\mathrm{Re}}(\vec{r})+i n_{\mathrm{Im}}(\vec{r})$ is the non-Hermitian potential, which is $n_{\mathrm{Re}}(\vec{r})$ for the real refractive index profile and $n_{\mathrm{Im}}(\vec{r})$ for the corresponding imaginary part of the potential obtained from the local HT with sink directionality.

We numerically solve Eq. (1) using the split-step method for HT media with different restrictions. For the complex refractive-index profiles shown in Figs. 3(a)-3(d), the simulated steady-state field distributions are provided in Fig. 5 , where a Gaussian source is initially placed at an arbitrary position within the modified structure, as shown in Fig. 5(ai). The system shows initially the transients as depicted in Fig. 5(aii), but eventually the field is efficiently concentrated around the center in Fig. 5(aiii), due to the sink directionality. The final localized states for different restrictions are shown in Fig. 5(b)-(d) (see the movie within the Supplemental Material [25] for the evolution of the field in the HT medium with different restrictions).

\section{CONCLUSIONS}

We propose a Restricted Hilbert transform for the generation of feasible systems to manage arbitrary field flows in higher-dimensional non-Hermitian systems under a realistic parameter space. In particular, we study restrictions of different dimensionalities from 2D to $1 \mathrm{D}$ and $0 \mathrm{D}$ to achieve the refractive indices for locally non-Hermitian systems within desired ranges. The procedure provides substantial control over the chosen index values compared with the conventional spatial Kramers-Kronig relation, and the constructed index profiles can be experimentally realized with a limited collection of realistic materials by locally tuning the real and imaginary parts of dielectric media. It is worth mentioning that a discrete HT requires a minimum of three different materials and three unaligned points on a susceptibility complex plane for realization. It also offers a general design strategy to implement any desired field configuration in a broad class of non-Hermitian systems conceptually different from existing coordinate transformation approaches [26-29]. The proposed theory opens up possibilities of practically realizing the wave dynamics of linear and nonlinear physical systems based on engineered HT media with realistic index and gain-loss values. 


\section{ACKNOWLEDGMENTS}

The work described here is partially supported by King Abdullah University of Science and Technology (KAUST) Office of Sponsored Research (OSR) under Grant No. OSR-2016-CRG5-2950, KAUST Baseline Research Fund BAS/1/1626-01-01, and NATO SPS research Grant No: 985048. K.S. acknowledges funding from the European Social Fund (Grant No. 09.3.3-LMT-K712-17- 0016) under grant agreement with the Research Council of Lithuania (LMTLT).

[1] J. D. Jackson, Classical Electrodynamics, 3rd Edition (Wiley, New York, 1999).

[2] S. A. R. Horsley, M. Artoni, and G. C. La Rocca, Spatial Kramers-Kronig relations and the reflection of waves, Nat. Photonics 9, 436 (2015).

[3] S. Longhi, Half-spectral unidirectional invisibility in nonHermitian periodic optical structures, Opt. Lett. 40, 5694 (2015).

[4] S. Longhi, Wave reflection in dielectric media obeying spatial Kramers-Kronig relations, Europhys. Lett. 112, 64001 (2015).

[5] S. A. R. Horsley, C. G. King, and T. G. Philbin, Wave propagation in complex coordinates, J. Opt. 18, 044016 (2016).

[6] Z. Hayran, R. Herrero, M. Botey, H. Kurt, and K. Staliunas, Invisibility on demand based on a generalized Hilbert transform, Phys. Rev. A 98, 013822 (2018).

[7] Z. Hayran, R. Herrero, M. Botey, H. Kurt, and K. Staliunas, All-Dielectric self-cloaked structures, ACS Photonics 5, 2068 (2018).

[8] K. G. Makris, R. El-Ganainy, D. N. Christodoulides, and Z. H. Musslimani, Beam Dynamics in PT Symmetric Optical Lattices, Phys. Rev. Lett. 100, 103904 (2008).

[9] A. Guo, G. J. Salamo, D. Duchesne, R. Morandotti, M. Volatier-Ravat, V. Aimez, G. A. Siviloglou, and D. N. Christodoulides, Observation of PT-Symmetry Breaking in Complex Optical Potentials, Phys. Rev. Lett. 103, 093902 (2009).

[10] C. E. Ruter, K. G. Makris, R. El-Ganainy, D. N. Christodoulides, M. Segev, and D. Kip, Observation of parity-time symmetry in optics, Nat. Phys. 6, 192 (2010).

[11] S. Longhi, Bloch Oscillations in Complex Crystals with PT Symmetry, Phys. Rev. Lett. 103, 123601 (2009).

[12] Z. Lin, H. Ramezani, T. Eichelkraut, T. Kottos, H. Cao, and D. N. Christodoulides, Unidirectional Invisibility Induced by PT-Symmetric Periodic Structures, Phys. Rev. Lett. 106, 213901 (2011).

[13] Y. D. Chong, L. Ge, and A. D. Stone, PT-symmetry Breaking and Laser Absorber Modes in Optical Scattering Systems, Phys. Rev. Lett. 106, 093902 (2012).
[14] L. Feng, Y.-L. Xu, W. S. Fegadolli, M.-H. Lu, J. E. Oliveira, V. R. Almeida, Y.-F. Chen, and A. Scherer, Experimental demonstration of a unidirectional reflectionless parity-time metamaterial at optical frequencies, Nat. Mater. 12, 108 (2012).

[15] B. Peng, S. K. Ozdemir, F. Lei, F. Monifi, M. Gianfreda, G. L. Long, S. Fan, F. Nori, C. M. Bender, and L. Yang, Parity-time-symmetric whispering-gallery microcavitie, Nat. Phys. 10, 394 (2014).

[16] L. Feng, R. El-Ganainy, and L. Ge, Non-Hermitian photonics based on parity-time symmetry, Nat. Photonics 11, 752 (2017).

[17] K. G. Makris, A. Brandstötter, P. Ambichl, Z. H. Musslimani, and S. Rotter, Wave propagation through disordered media without backscattering and intensity variations, Light Sci. Appl. 6, e17035 (2017).

[18] R. El-Ganainy, K. G. Makris, M. Khajavikhan, Z. H. Musslimani, S. Rotter, and D. N. Christodoulides, NonHermitian physics and PT symmetry, Nat. Phys. 14, 11 (2018).

[19] W. W. Ahmed, R. Herrero, M. Botey, and K. Staliunas, Locally parity-time-symmetric and globally paritysymmetric systems, Phys. Rev. A 94, 053819 (2016).

[20] W. W. Ahmed, R. Herrero, M. Botey, Z. Hayran, H. Kurt, and K. Staliunas, Directionality fields generated by a local Hilbert transform, Phys. Rev. A 97, 033824 (2018).

[21] W. Jiang, Y. Ma, Y. Jun, G. Yin, W. Wu, and W. He, Deformable broadband metamaterial absorbers engineered with an analytical spatial Kramers-Kronig permittivity profile, Laser Photon. Rev. 11, 1600253 (2017).

[22] D. Ye, C. Cao, T. Zhou, J. Huangpu, G. Zheng, and L. Ran, Observation of reflectionless absorption due to spatial Kramers-Kronig profile, Nat. Commun. 8, 51 (2017).

[23] A. Thelen, Design of Optical Interference Coatings (McGraw-Hill, New York, 1989).

[24] T. Tolenis, L. Grinevičiūtè, R. Buzelis, L. Smalakys, E. Pupka, S. Melnikas, A. Selskis, R. Drazdys, and A. Melninkaitis, Sculptured anti-reflection coatings for high power lasers, Opt. Mater. Express 7, 1249 (2017).

[25] See the Supplemental Material at http://link.aps.org/supple mental/10.1103/PhysRevApplied.14.044010 for different possible restriction strategies in the proposed procedure, realization of Restricted HT with random background patterns and lossy materials, and implementation of HT beyond the paraxial limit. The movie demonstrates the field evolution for both unrestricted and Restricted HT cases with sink directionality, and the probe field is normalized at every instant during evolution for the representation.

[26] J. B. Pendry, D. Schurig, and D. R. Smith, Controlling electromagnetic fields, Science 312, 1780 (2006).

[27] U. Leonhardt, Optical conformal mapping, Science 312, 1777 (2006).

[28] H. Y. Chen, C. T. Chen, and P. Sheng, Transformation optics and metamaterials, Nat. Mater. 9, 387 (2010).

[29] L. Xu and H. Chen, Conformal transformation optics, Nat. Photonics 9, 15 (2015). 